MATEC Web of Conferences 6, 05007 (2013)

DOI: $10.1051 /$ matecconf/20130605007

(C) Owned by the authors, published by EDP Sciences, 2013

\title{
On explicit modeling of polypropylene fiber effects on hydro-thermal behavior of heated concrete
}

\author{
V.H. $\operatorname{Tran}^{1}$, F. Meftah ${ }^{1}$, L. Izoret ${ }^{2}$ and M. Behloul ${ }^{3}$ \\ 1 LGCGM - INSA Rennes, Rennes, France \\ 2 ATILH, Paris-La-Defense, France \\ ${ }^{3}$ Lafarge Ciments Distribution, Saint-Cloud, France
}

\begin{abstract}
This work focuses on the evaluation of the probability of percolation of polypropylene fibers (PPF) in creating a permeable network within heated concreted due to temperature induced degradation of fibers. Therefore, the intrinsic permeability supply of heated concrete containing PPF is accordingly estimated by a homogenization technique applied to the $3 \mathrm{D}$ heterogeneous microstructure.
\end{abstract}

\section{INTRODUCTION}

It is well established now that adding polypropylene fibers (PPF) to HPC and UHPC may reduce spalling occurrence during fire [1,2]. In several experimental studies [3, 4], it has been observed a significant increase of permeability, in the temperature interval from $150^{\circ} \mathrm{C}$ to $250^{\circ} \mathrm{C}$, of heated concrete containing PPF. This sharp increase contrasts with a more progressive increase, with temperature, of permeability of concrete not containing PPF. It is worth noting that permeability increase leads to a reduction of internal pore pressures; either gas pressure in dried zones or liquid pressure in full saturated zones (due to water clog). Indeed, low gas pressures have been measured when PPF are added [2].

The increase of permeability of heated concrete is assumed to be a key parameter of PPF performance to reduce spalling risk $[1,2]$. Nevertheless, there is no consensus on mechanisms which lead to the increase of permeability. A first plausible mechanism [5] is that PPF weaken the cement paste which favors the early occurrence of a connected network of cracks. These latter could initiate at either aggregates or fibers and thus connect paste-aggregate transitional zones with endemic high porosity. A second plausible mechanism relates the increase of permeability to the additional connected voids induced by PPF transformation that starts when temperature exceeds a threshold value above the melting point (around $160^{\circ} \mathrm{C}$ ) up to the full degradation of PPF (around $300^{\circ} \mathrm{C}$ ). Fiber degradation releases fiber beds (within cement paste) that percolate and thus create additional porous network.

Probably, both mechanisms co-exist and the challenge is to evaluate quantitatively their relative contributions. In this work, the second mechanism, discussed above, is considered. The aim is to estimate permeability increase based on a limited set of input data: fiber content, fiber slenderness and temperature interval of fiber activation (ranging from melting to degradation points) appear to be three influent factors that may be used as design parameters of PPF-concrete.

For this purpose, a numerical model is developed in order to estimate, during heating, permeability evolution of concretes containing PPF. The estimation method is based on the analysis of percolation of clusters of fibers randomly generated in the material. The analysis (either 2D or 3D) accounts for the heterogeneity of the material, that is, fibers are randomly generated within the cement paste which fills space between aggregates themselves randomly generated according to their gradation. Accordingly, an existing thermo-hygral finite element model [6] is extended to take into account explicitly effects of PPF on heat and mass transfer in heated concrete.

This is an Open Access article distributed under the terms of the Creative Commons Attribution License 2.0, which permits unrestricted use, distribution, and reproduction in any medium, provided the original work is properly cited. 


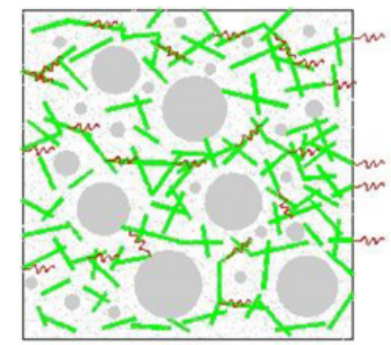

(a)

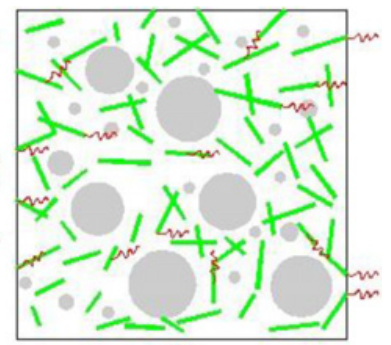

(b)

Figure 1. Schematic of Voigt (a) and Reuss (b) mass transfer mechanisms.

In this work, some experimental results, available in the literature, are investigated using the developed cluster percolation model. On one hand, experimentally measured permeability evolutions are compared to estimated ones. On the other hand, different PPF additions are evaluated in relation to the theoretical threshold content (that ensures network percolation of PPF) and their efficiency in limiting or not spalling.

\section{PERMEABILITY OF HEATED PPF-CONCRETE}

The permeability tensor $\mathbf{K}_{f c}$ of concrete containing PPF is determined from micromechanical consideration using the upper and lower estimation bounds of Voigt $\mathbf{K}_{f c}^{V}$ and Reuss $\mathbf{K}_{f c}^{R}$ :

$$
\mathbf{K}_{f c}=p_{f} \mathbf{K}_{f c}^{V}+\left(1-p_{f}\right) \mathbf{K}_{f c}^{R}
$$

where $p_{f}\left(c_{f}, \varepsilon_{f}\right)$ is the probability of percolation of the space released by the network of PPF beds due to thermal degradation of PPF. This probability is considered to depend on the volume fraction $c_{f}$ and the aspect ratio (slenderness) $\varepsilon_{f}$ of the considered fiber, with:

$$
\varepsilon_{f}=\frac{L_{f}}{d_{f}}
$$

where $L_{f}$ and $d_{f}$ are, respectively, its large and small characteristic dimensions.

The Voigt model (Fig. 1a) corresponds to the situation in which fibers have totally percolate $\left(p_{f}=1\right)$ allowing for separate tranfer within the percolated network. In this case, the permeability tensor writes [7]:

$$
\mathbf{K}_{f c}^{V}=c_{f} \mathbf{K}_{f}+\left(1-c_{f}\right) \mathbf{K}_{c} .
$$

The Reuss model (Fig. 1b) corresponds to the case where the medium alternates mass tranfer within the porous matrix and the fibers network:

$$
\mathbf{K}_{f c}^{R}=\left(c_{f} \mathbf{K}_{f}^{-1}+\left(1-c_{f}\right) \mathbf{K}_{c}^{-1}\right)^{-1} .
$$

In both cases, $\mathbf{K}_{c}$ is the istropic permeability tensor of the porous matrix:

$$
\mathbf{K}_{c}=K(T) \boldsymbol{\delta}
$$

where $\delta$ is the second order unit tensor and $K(T)$ is the temperature dependent intrisic permability given phenomenologically by [8]:

$$
K(T)=K_{0} \cdot 10^{A_{T}\left(T-T_{0}\right)}
$$

$K_{0}$ being the initial permeability at the reference temperature $T_{0}$. 
With regard to additional permeability due PPF addition, it is assumed that temperature induced degradation of PPF leads to a directional Poiseuille transport of fluids along each fiber bed. Considering cylindrical fibers with diameter $d_{f}$, the directional permeability reads:

$$
K_{f}(T)=\frac{d_{f}^{2}}{32} h_{f}(T)
$$

which allows to expression the tensor of anisotropic permeability due to fiber as:

$$
\mathbf{K}_{f}=K_{f}(T) \sum_{i=1}^{N_{f}} \vec{n}^{(i)} \otimes \vec{n}^{(i)}
$$

where $N_{f}$ is the number of fibers, $\vec{n}^{(i)}$ the unit vector giving the orientation of a fiber $i$ and $\otimes$ stands for the tensor product.

Furthermore, the function $h_{f}(T)$ in equation (7) ranges from zero at the melting temperature $T_{0}^{f}$ to one at the temperature $T_{1}^{f}$ of full degradation of the PPF. This means that the permeability supply due to PPF degradation is assumed to be progressive since it is observed [5] a progressive release of PPF beds during heating. Fore sake of simplicity a linear evolution of is considered is this work. The temperature interval $\left(T_{0}^{f}, T_{1}^{f}\right)$ is called the thermal activation interval of the fiber and is considered as the third input in the proposed model. It is worth noting that one aim of this work is to limit the set of input data to fit as much as possible some accessible design parameters of PPF-concrete.

\section{PERCOLATION PROBABILITY OF PPF NETWORK}

In equation (1), there is a need to evaluate the probability of percolation $p_{f}$ of the network of voids released by fiber beds within the cement paste matrix. This is performed thanks to a developed numerical model based on a statistical cluster sampling approach. This later consists of generating a cluster of $N_{e}$ elements, each element contains $N_{e}^{f}$ fibers that are randomly generated within a simulation cell that has a size $L_{e}$ larger than fiber length $L_{f}$ in order to fulfill the requirement $L_{f} \ll L_{e}$ of scale separation [7]. Some preliminary simulations are performed by varying the ratio $L_{e} / L_{f}$ up to attain an asymptotic value of $p_{f}$ which allows to identify the minimum value of $L_{e} / L_{f}$ that makes the value of $p_{f}$ objective.

The random generation of fibers within each cluster-element is based on given probability density functions (PDF) for the coordinates $\left(x_{0}^{f}, y_{0}^{f}, z_{0}^{f}\right)$ of fiber centroid and the angles $\left(\theta_{0}^{f}, \varphi_{0}^{f}\right)$ of orientation of the fiber. No correlation is assumed, neither spatial for a given geometric parameter $\bullet_{0}^{f}$ nor between these geometric parameters.

For each cluster-element randomly generated for a fixed $N_{e}^{f}$, an analysis is performed of the existence or not of a connected path, belonging to fibers and which crosses the simulation cell form one side to the opposite side. This analysis is based on the algorithm proposed by Vieillard-Baron [11]. It consists of analyzing the overlapping (or at the limit the contact) between fibers based on an ad hoc criterion. For this aim, cylindrical fibers are represented by equivalent ellipsoids for which such an overlapping criterion exists $[9,10]$. Figure 2 illustrates the identification process of connected paths (in red) up to percolation from one side to the opposite side of a simulation unit-cell containing randomly generated fibers (ellipses in 2D and ellipsoids en 3D).

If a percolated path is detected then the cluster-element is added to the set of $N_{e}^{c}$ cluster-elements exhibiting percolation. Therefore, the probability of percolation is statistically obtained by:

$$
p_{f}\left(c_{f}, \varepsilon_{f}\right)=\frac{N_{e}^{c}}{N_{e}} .
$$

In equation (9), $c_{f}$ is the effective volume fraction, that is, the volume fraction that account for the overlapping of randomly generated fibers. The common volume between several overlapping fibers gets to count once when determining the effective volume fraction of fibers. 


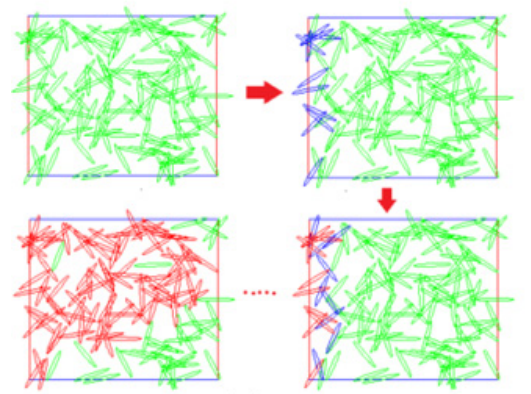

(a)

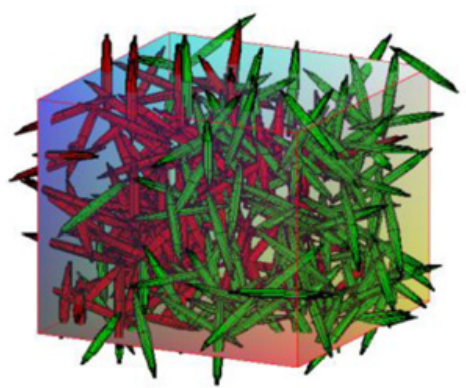

(b)

Figure 2. Illustration of the percolation algorithm in 2D.

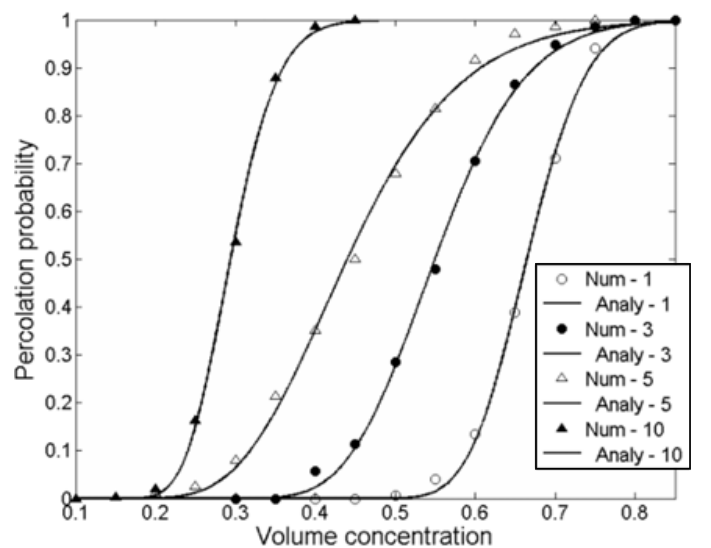

(a)

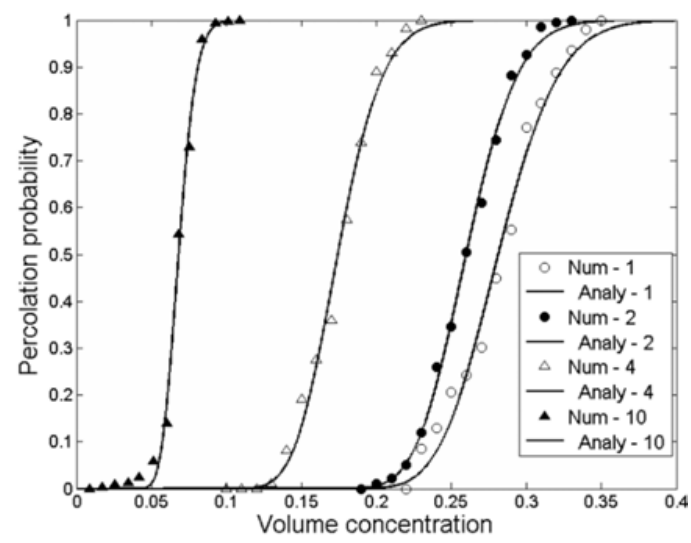

(b)

Figure 3. Percolation probability in 2D (a) and in 3D (b): discrete versus fitted.

Finally, the discrete values of $p_{f}$ corresponding to a set of values of $c_{f}$ for a fixed $\varepsilon_{f}$ are fitted using a cumulative distribution function $(\mathrm{CDF})$ assumed to correspond to a log-normal density [13]:

$$
p_{f} \approx \frac{1}{2}\left(1+\operatorname{erf}\left(\frac{\ln \left(c_{f}\right)-\mu}{\sigma \sqrt{2}}\right)\right)
$$

where $\mu$ is the mean and $\sigma$ the standard deviation.

Figures 3 and 4 illustrate results obtained by the developed numerical tool in 2D/3D configurations with $N_{e}=500$ and some selected aspect ratios of fibers: $\varepsilon_{f}=1,3,5,10$ in $2 \mathrm{D}$ and $\varepsilon_{f}=1,2,4,10$. Figure 3 shows numerical discrete values of $p_{f}$ according to Eq. (9) versus the analytical fitting function of Eq. (10). Figure 4 show a good agreement of the developed tool with results obtain in $[9,10]$.

\section{AGGREGATES INDUCED HETEROGENEITY}

The developed algorithm developed in the previous section is extended to take into account of the material (concrete) heterogeneity due to aggregates. The analysis is limited to coarse aggregates that have diameters larger than $5 \mathrm{~mm}$. Therefore, fine aggregates and cement paste are considered to form the matrix within which PPFs are randomly generated. That is, PPF are not allowed to overlap with 


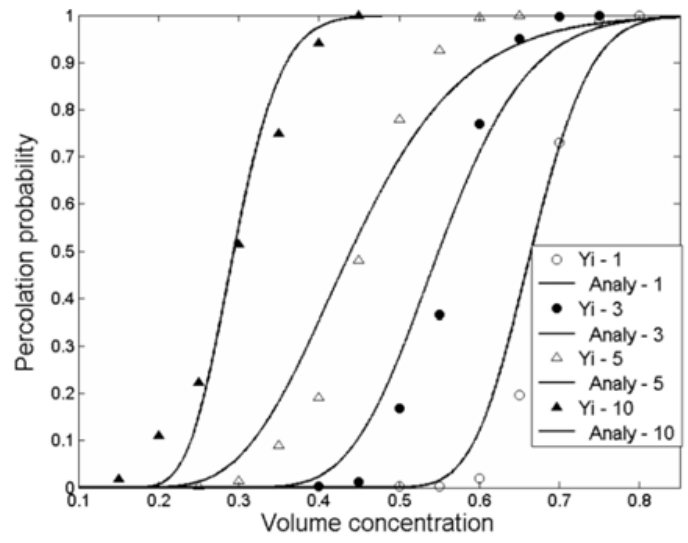

(a)

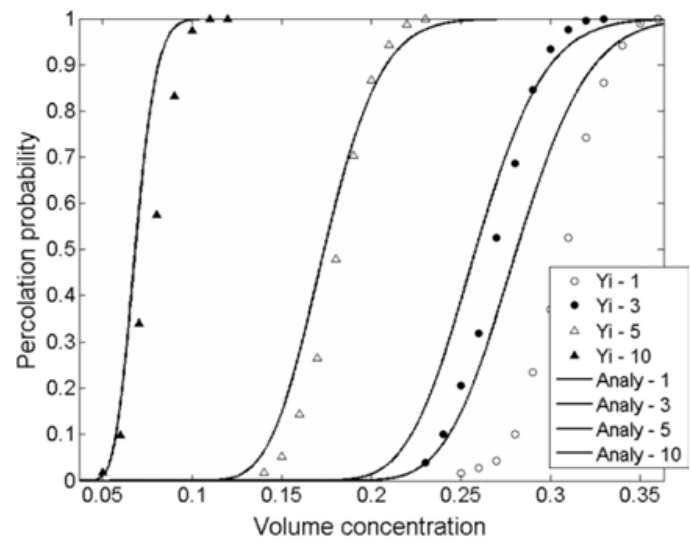

(b)

Figure 4. Percolation probability: confrontation with Yi \& al results $[9,10]$ in 2D (a) and 3D (b).

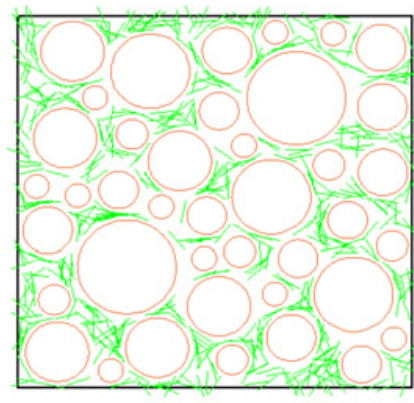

(a)

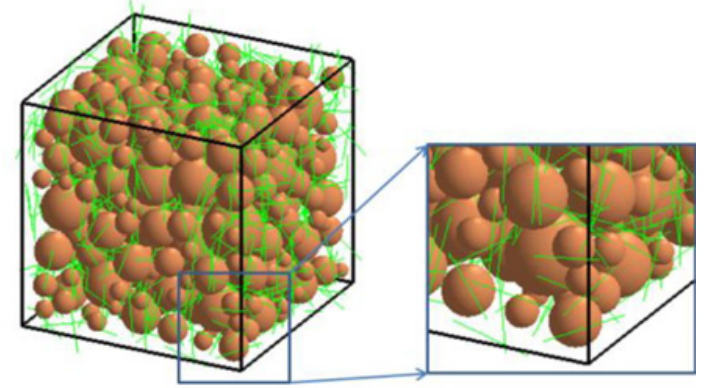

(b)

Figure 5. Idealized microstructure of a PPF-concrete: in 2D (a) and in 3D (b).

already generated aggregates. These later are represented (idealized) by circles (2D) or spheres (3D) with a set of diameters corresponding to sieve openings used for determining aggregate grading. For each class of diameter a volume fraction is identified in order to determine the number of idealized aggregates belonging to this class.

Figure 5 represents an illustration of 2D/3D idealized microstructures of concrete containing PPF which have been generated in order to estimate the probability of percolation $p_{f}$ of the PPF network in the heterogeneous case.

\section{PARAMETRIC STUDY}

In the developed cluster base model of percolation, the cylindrical PPF is represented by equivalent ellipsoid of revolution in order to use an existing analytical criterion of overlapping between fibers. In this section, a parametric study is performed with regard to the repercussion of the choice of the equivalence criterion on the estimated $p_{f}\left(c_{f}, \varepsilon_{f}\right)$. The following four criteria of equivalent between a cylinder and an ellipsoid are adopted:

- Equivalence criterion 1 (EC1): $\left(L_{f}\right)_{e}=\left(L_{f}\right)_{c}$ and $\left(d_{f}\right)_{e}=\left(d_{f}\right)_{c}$ which leads to $\left(\varepsilon_{f}\right)_{e}=\left(\varepsilon_{f}\right)_{c}$ and to an increase of the number of fibers $N_{f}$. 


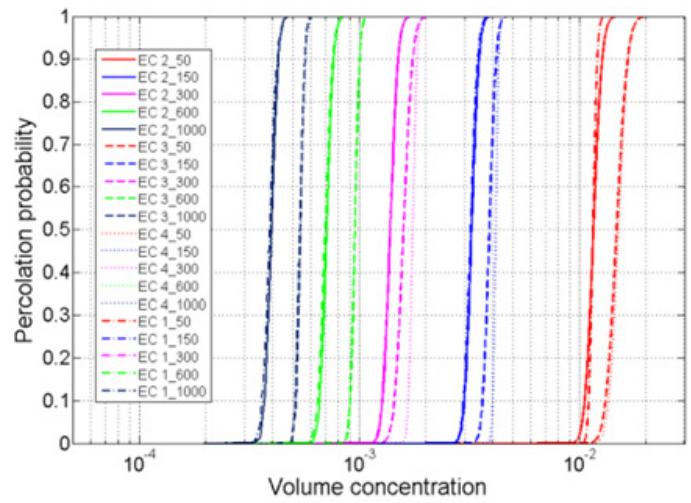

(a)

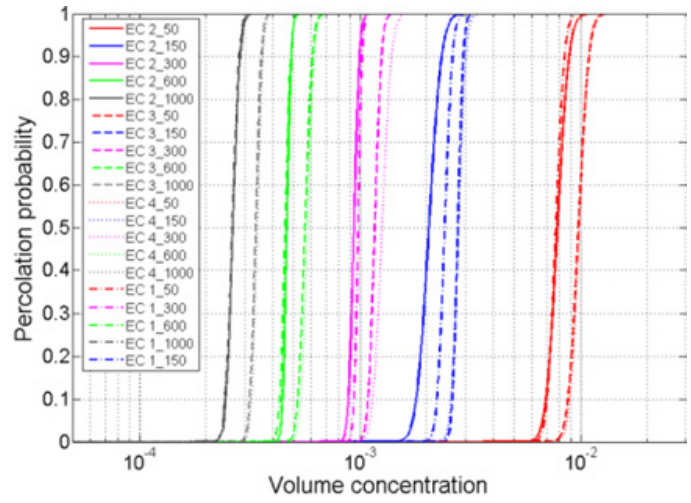

(b)

Figure 6. Estimated $p_{f}$ versus $c_{f}$ : homogeneous (a) and heterogeneous (b) cases.

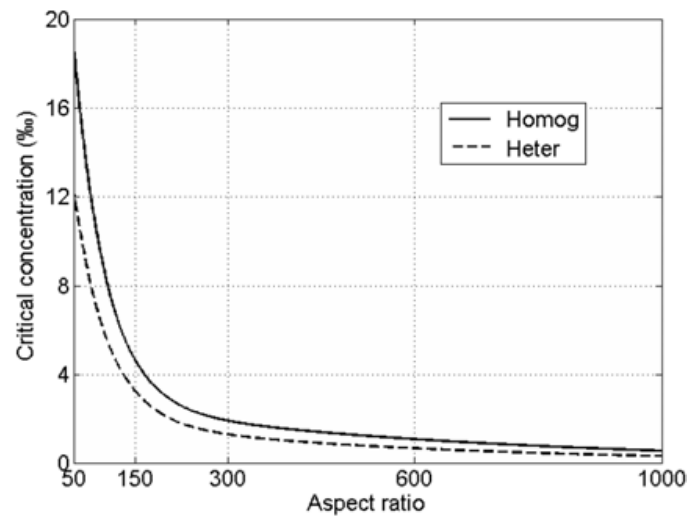

Figure 7. Evolution of critical concentration $c_{f}^{c}$ of PPF with fiber slenderness.

- Equivalence criterion 2 (EC2): $\left(V_{f}\right)_{e}=\left(V_{f}\right)_{c},\left(L_{f}\right)_{e}=\alpha_{f}\left(L_{f}\right)_{c}$ and $\left(d_{f}\right)_{e}=\alpha_{f}\left(d_{f}\right)_{c}$ which leads to $\left(\varepsilon_{f}\right)_{e}=\left(\varepsilon_{f}\right)_{c}$.

- Equivalence criterion 3 (EC3): $\left(V_{f}\right)_{e}=\left(V_{f}\right)_{c},\left(L_{f}\right)_{e}=\left(L_{f}\right)_{c}$ and $\left(d_{f}\right)_{e}=\alpha_{f}\left(d_{f}\right)_{c}$ which leads to $\left(\varepsilon_{f}\right)_{e}=\alpha_{f}^{-1}\left(\varepsilon_{f}\right)_{c}$.

- Equivalence criterion 4 (EC4): $\left(\bar{S}_{f}\right)_{e}=\left(\bar{S}_{f}\right)_{c},\left(L_{f}\right)_{e}=\left(L_{f}\right)_{c}$ and $\left(d_{f}\right)_{e}=\alpha_{f}\left(d_{f}\right)_{c}$ which leads to $\left(\varepsilon_{f}\right)_{e}=\left(\varepsilon_{f}\right)_{c}$.

where $(\bullet)_{c}$ refers to a quantifiy characterizing the original cylindrical fiber while $(\bullet)_{e}$ refers to the quantity corresponding the equivalent ellipsoid. Moreover, $V_{f}$ is the volume of the fiber and $\bar{S}_{f}$ its specific surface. The coefficient $\alpha_{f}$ is identified from the requirement $\left(V_{f}\right)_{e}=\left(V_{f}\right)_{c}$ or $\left(\bar{S}_{f}\right)_{e}=\left(\bar{S}_{f}\right)_{c}$ for the considered EC. Note that if for EC4 one adopts $\left(L_{f}\right)_{e}=\alpha_{f}\left(L_{f}\right)_{c}$ than $\alpha_{f}$ will have values almost equal to that of EC2.

Five aspect ratios $\varepsilon_{f}$ are considered: 50, 150, 300, 600 and 1000 which covers used PPF in some works found in the literature $[3,4,12]$. Figure 7 gives the estimated $p_{f}\left(c_{f}, \varepsilon_{f}\right)$ in both homogeneous and heterogeneous (3D) cases for analyzed PPF-concretes.

With regard to the choice of equivalence criterion, similar estimations are obtained, on one hand, by EC1 and EC2 and, on the other hand, by EC3 and EC4. The first family of EC gives an upper bound 
IWCS 2013

Table 1. Spalling occurrence for different concrete mixes versus PPF-critical-content.

\begin{tabular}{|l|c|c|c|r|c|c|l|}
\hline Ref. & Type & $L_{f}(\mathrm{~mm})$ & $d_{f}(\mu m)$ & $\varepsilon_{f}(-)$ & PPF-mc $\left(\mathrm{kg} \cdot \mathrm{m}^{-3}\right)$ & PPF-cc $\left(\mathrm{kg} \cdot \mathrm{m}^{-3}\right)$ & Spalling occurrence \\
\hline$[3]$ & M-F & 6 & 18 & 333 & 1.5 & 1.160 & Not reported \\
\hline$[4]$ & M-F & 12 & 18 & 667 & 2.0 & 0.574 & No \\
\hline$[12]$ & M-F & 18 & 18 & 1000 & 0.0 & 0.377 & Up to 6 cm depth \\
\hline$[12]$ & M-F & 18 & 18 & 1000 & 0.9 & 0.377 & No \\
\hline$[12]$ & M-F & 18 & 18 & 1000 & 1.2 & 0.377 & No \\
\hline$[12]$ & S-F & 6 & 50 & 120 & 1.5 & 4.500 & Up to 6 cm depth \\
\hline$[12]$ & F & 19 & 50 & 380 & 1.5 & 1.000 & Less than 1 cm depth \\
\hline
\end{tabular}

while the second one gives a lower bound of the estimated fiber concentration $c_{f}$ for a given target value $p_{f}$ of the probability of percolation of the PPF network.

Moreover, the homogeneous estimation appears, with respect to heterogeneous case, to overestimate the PPF concentration which is needed to achieve a target value of probability of percolation. In other words, the homogeneous case is conservative for estimating PPF content for concrete mix design.

A critical PPF concentration $c_{f}^{c}$ can therefore be introduced for design of concrete mix containing PPF: the concrete mix should have a PPF content larger than the critical value. The critical concentration may be defined, for instance, as the minimal value of $c_{f}$ that achieves $p_{f}\left(c_{f}^{c}=\min \left(c_{f}\right), \varepsilon_{f}\right)=1$. Figure 7 gives the evolution of this critical PPF concentration $c_{f}^{c}$ with the slenderness of the fiber for the considered PPF-concrete mixes.

\section{PPF-CONTENT VERSUS SPALLING}

In this section, the PPF-critical-content (PPF-cc), obtained from $c_{f}^{c}$ as the critical mass of PPF per unit volume of concrete, is compared to the PPF-mix-content (PPF-mc) of some PPF-concrete mixes available in the literature. For some of these concretes, spalling occurrence or not has been reported. The available data and the comparison are reported in table 1 in which three types of PPF are considered: monofilament (M-F), semi-fibrillated (S-F) and fibrillated (F).

This table shows that no spalling is reported when PPF-mc is too much larger than the estimated PPF-cc, this later being the threshold at which a probably full percolation of the PPF network is achieved. Conversely, in the case where PPF-mc is zero or too much smaller (see case 6 in the table with S-F PPF) than the PPF-cc a severe occurrence of spalling is observed. Finally, a slight spalling is reported when PPF-cc and PPF-mc have close values.

\section{CONCLUSION}

In this work, effect of PPF on heated concrete are investigated based on an explicit representation of the microstructure of concrete containing PPF. The performance of PPF addition is assessed through the evaluation of the probability of percolation of their network. This probability is then introduced as a key parameter for estimating the supply of permeability of heated PPF-concrete due to fiber degradation with temperature. Moreover, the probability of percolation has lead to the identification of a critical value of PPF concentration that achieves full percolation of fibers. When the PPF concentration is much larger, respectively, lower than this critical threshold value then no, respectively, a significant spalling is reported on some tested concrete mixes. This suggests that the critical PPF-content may be adopted as a primary design parameter for PPF-concrete mixes. 


\section{MATEC Web of Conferences}

\section{References}

[1] Bentz D.P., Fibers percolation, and spalling of high-performance concrete. ACI Materials Journal, 97 (3), 351-359, 2000.

[2] Kalifa P., Chene G., Galle C., High-temperature behaviour of HPC with polypropylene fibres: From spalling to microstructure. CCR, 31(10), 1487-1499, 2001.

[3] Zeiml M., Leithner D., Lackner R., Mang H.A., How do polypropylene fibers improve the spalling behavior of in-situ concrete? CCR, 36(5), 929-942, 2006.

[4] Mindeguia J. C., Contribution expérimentale à la compréhension des risques d'instabilité thermique des bétons. Ph.D Thesis (in French). Université de Pau, 2009.

[5] Pistol K., Weise F., Meng B., Polypropylen-Fasern in Hochleistungsbetonen. Beton-und Stahlbetonbau, 107(7), 476-483, 2012.

[6] Meftah F., Dal-Pont S., Schrefler B.A., A three-dimensional staggered finite element approach for random parametric modeling of thermo-hygral coupled phenomena in porous media. Int. J. for Num. and Anal. Meth. in Geomechanics, 36(5), 574-596, 2012.

[7] Mura T., Micromechanics of defects in solids. Kluwer Academic Pub., 1987.

[8] Gawin D., Majorana C.E., Schrefler B.A., Numerical analysis of hygro-thermal behaviour and damage of concrete at high temperature. Mechanics of Cohesive-frictional Materials, 4(1), 37-74, 1999.

[9] Yi Y.B., Sastry A.M., Analytical approximation of the two-dimensional percolation threshold for fields of overlapping ellipses. Physical Review E, 66(6), 066130, 2002.

[10] Yi Y.B., Sastry A.M.. Analytical approximation of the percolation threshold for overlapping ellipsoids of revolution. Proc. Roy. Soc. Series A: Math., Phys. Eng. Sci., 460(2048), 2353-2380, 2004.

[11] Vieillard-Baron J., Phase transitions of the classical hard-ellipse system. J. Chem. Phys., 56, 4729-4744, 1972.

[12] Étude Feu-Béton, Final report (in French), Research project on the fire behavior of concrete, CERIB-ATILH, 2006.

[13] Bogaert P., Probabilités pour scientifiques et ingénieurs: Introduction au calcul des probabilités. De Boeck, 2005. 